\title{
Case Report: Primaquine Failure for Radical Cure of Plasmodium vivax Malaria in Gambella, Ethiopia
}

\author{
Awash Teklehaimanot, ${ }^{1 \star}$ Hailay Teklehaimanot, ${ }^{1}$ Abeba Girmay, ${ }^{2}$ and Adugna Woyessa ${ }^{3}$ \\ ${ }^{1}$ Centers for National Health Development Ethiopia, Addis Ababa, Ethiopia; ${ }^{2}$ Laboratory Department, Saint Paulos Hospital, Millennium Collage, \\ Addis Ababa, Ethiopia; ${ }^{3}$ National Research Institute of Health, Addis Ababa, Ethiopia
}

\begin{abstract}
Failures of primaquine for the treatment of relapsed Plasmodium vivax malaria is a serious challenge to malaria elimination in Ethiopia, where $P$. vivax accounts for up to $40 \%$ of malaria infections. We report here occurrence of a total of 15 episodes of primaquine treatment failure for radical cure in three historical $P$. vivax malaria patients from Gambella, Ethiopia, during 8-16 months of follow-up in 1985-1987. The total primaquine doses received were 17.5 $\mathrm{mg} / \mathrm{kg}, 25.8 \mathrm{mg} / \mathrm{kg}$, and $35.8 \mathrm{mg} / \mathrm{kg}$, respectively. These total doses are much higher than in previous reports of patients with treatment failure in Ethiopia and East Africa. The possibility of new infection was excluded for these cases as the treatment and follow-up were carried out in Addis Ababa, a malaria-free city. Recrudescences were unlikely, considering the short duration pattern of the recurrences. The cytochrome P450 2D6 (CYP2D6) status for these patients is unknown, but polymorphisms have been described in Ethiopia and may have contributed to primaquine treatment failures. It is suggested that further studies be carried out in Ethiopia to determine the prevalence and distribution of primaquine treatment failures in different ethnic groups, considering the impact of CYP2D6 polymorphisms and the potential value of increasing the primaquine dose to avoid relapse.
\end{abstract}

\section{INTRODUCTION}

Approximately $68 \%$ of the population of Ethiopia live in areas at risk of malaria infection. ${ }^{1}$ According to the health and health-related indicators (2007EC) of the Federal Ministry of Health, malaria is one of the top 10 causes of morbidity and mortality in Ethiopia. ${ }^{2}$ Both Plasmodium falciparum and Plasmodium vivax are co-endemic in Ethiopia. With an estimated 3-5 million cases per year, $P$. vivax accounts for up to $40 \%$ of all malaria infections in Ethiopia. ${ }^{1}$ Plasmodium vivax is characterized by the development of dormant stages of parasites inside hepatic cells, which are called hypnozoites. ${ }^{3,4}$ Hypnozoites trigger relapsing episodes of blood-stage infections with different periodicity, for example, the relapses in Southeast Asia are characterized by early and frequent episodes, indicating the need for higher dosing of primaquine therapy. 5,6 The hypnozoite reservoirs present a challenge for malaria control and elimination, as the carriers are asymptomatic., ${ }^{3,4,7}$

Embracing the African Malaria Strategy, the National Malaria Control Program (NMCP) has launched a first phase of elimination program in selected districts. ${ }^{8}$ Radical cure of $P$. vivax is critical for the elimination of $P$. vivax, and the country adopted primaquine based on the WHO recommendation of $15 \mathrm{mg}$ base daily for 14 days. ${ }^{9}$ However, some studies have shown that the standard WHO-recommended primaquine dose has failed to radically cure $P$. vivax infections in Ethiopia and East Africa. ${ }^{10,11}$ Thus, there is a need to establish the primaquine dose that failed to prevent relapse of $P$. vivax infections.

In this case series, we describe three historical cases from the 1980s with a total of 15 episodes of primaquine treatment failure for radical cure. The objective of the case series is to characterize relapsing $P$. vivax infections and document the primaquine dose that failed to prevent relapse of $P$. vivax infections. The specific objectives include the following: to retrospectively analyze data collected from routine healthcare

*Address correspondence to Awash Teklehaimanot, Center for National Health Development Ethiopia, PO Box 664, Addis Ababa 1250, Ethiopia. E-mail athbruk1@gmail.com services of three $P$. vivax-infected cases, describe the pattern of relapse characteristics of $P$. vivax, determine the dose of primaquine associated with relapse of $P$. vivax, understand the clinical and hematological effects associated with the use of primaquine for radical cure of $P$. vivax infections, and identify issues that should be considered in future research on effective dose of primaquine for the treatment of relapsing $P$. vivax in Ethiopia. A case series approach was used through compilation, analysis, and synthesis of medical records of the $P$. vivax cases. The medical records including clinical presentation, parasite counts, treatment dates and doses, blood chemistry tests, and outcome of treatment were retrieved and analyzed to achieve the objective of the case series. An official ethical approval to conduct case series analysis was obtained from the Ethiopian Public Health Institute Institutional Review Committee.

\section{STUDY SETTING}

Three permanent residents of Addis Ababa, a malaria-free capital city of Ethiopia, located at an altitude 2,355 m (7,726 feet) above sea level, had traveled to Gambella, a malariaendemic region in western Ethiopia, to provide community service of population resettlement scheme during 1985-1987. The program involved resettlement of people from overcrowded and drought-prone highland areas to underused fertile lowlands such as Gambella. With an average altitude of 300-500 m above sea level and annual rainfall of $120 \mathrm{~mm}$, Gambella is one of the few areas in Ethiopia that have an intense perennial malaria transmission. The three cases had stayed in Gambella malaria-endemic area for 2-11 months before they returned to Addis Ababa. The cases presented with fever at the National Malaria Laboratory Center, Ministry of Health, Addis Ababa, 4-49 days after their return to Addis Ababa. The center was set up by the Ministry of Health to provide free malaria diagnostic and treatment services to self-reporting patients. They were diagnosed with $P$. vivax mono-infection parasites and treated with standard dose of chloroquine followed by primaquine therapy. The cases were followed up from 8 to 16 months at the Malaria Laboratory 
Center in Addis Ababa, outside a transmission area, which provided an opportunity to document the pattern of relapse characteristics of $P$. vivax malaria.

\section{CASE PRESENTATION}

We report here clinical records of three Addis Ababa residents, designated as case 1,2 , and 3 , who had stayed in Gambella malaria-endemic area for 11, 2, and 3 months, respectively, before they returned to Addis Ababa. The three cases who reported that they were not ill during their stay in Gambella self-reported at the National Malaria Laboratory Center in Addis Ababa with fever between 1985 and 1986 seeking diagnosis and treatment services that are provided for free. Giemsa-stained blood smear examination of the cases revealed the presence of $P$. vivax mono-infection parasites. Details of the three cases are as follows.

Case 1 was a 38-year-old man weighing $64 \mathrm{~kg}$ who had stayed in Ubala, Gambella, for 11 months from October 1985 to August 1986. He was on regular weekly $300 \mathrm{mg}$ base chloroquine prophylaxis only for the first 8 months of his 11month stay in Gambella (Figure 1). He returned to Addis Ababa in September 1986 and reported with symptoms suggestive of malaria 49 days after his return to Addis Ababa. Case 1 experienced four relapsing attacks during an 8-month follow-up (Table 1). The first three relapses were of short interval with an average of 28 days and a range of 26-32 days, whereas the fourth relapse occurred at a 121-day interval.

Case 2 was a 34-year-old man weighing $60 \mathrm{~kg}$ who had stayed in Ubala, Gambella, for 2 months from July 1985 to August 1985 . He was on regular weekly 300 mg base chloroquine prophylaxis before his departure for Gambella, during his 2-month stay in Gambella, and for additional 4 weeks (September 1985) after his return to Addis Ababa in August 1985 (Figure 1). He reported with symptoms suggestive of malaria 47 days after his return to Addis Ababa and 22 days after his last chemoprophylaxis dose. Case 2 experienced five relapsing attacks during the 9-month follow-up (Table 2). The intervals of the four relapses were of short duration with an average interval of 26 days and a range of $27-40$ days. The fifth relapse occurred after an interval of 63 days.

Case 3 was a 52-year-old man weighing $52 \mathrm{~kg}$ who had stayed in Keto-Shebelle, Gambella, for 3 months (June 1986-August 1986) but did not take any malaria chemoprophylaxis for this trip. He returned to Addis Ababa at the end of August 1986 and reported with symptoms suggestive of malaria 4 days after his return to Addis Ababa (Figure 1). Case 3 had six relapsing attacks during 16 months of follow-up. The first four relapses occurred at an interval of 37 days and a range of 29-47 days, whereas the fifth and sixth relapses occurred at an interval of 99 days and 154 days, respectively (Table 3). Overall, 11 of the total 15 relapse attacks of the three cases had a short interval of about 1-month duration and the other four relapses occurred at an interval of 2 months to 5.1 months (Figure 1).

\section{TREATMENT AND OUTCOME}

The first attacks of each of the three cases were treated with a standard dose of $1,500 \mathrm{mg}$ base chloroquine over 3 days followed by a low-dose primaquine therapy of $15 \mathrm{mg}$ per day for 5 days based on national guidelines. Until a few years ago, the general practice of malaria control program in Ethiopia involved the use of 5-day primaquine therapy to treat $P$. vivax cases among permanent residents of endemic areas and mobile populations. The 5-day primaquine regimen for $P$. vivax malaria treatment protocol was also intended to target $P$. falciparum gametocytes, as the prevalence of $P$. falciparum and $P$. vivax mixed infections is very high in Ethiopia. Radical treatment was provided to those who live in malaria-free areas if they agree to comply with the 14-day primaquine treatment regimen. After the first attacks were managed, the three cases reported back to the same malaria center with complaints of malarial illness for a number of times with different intervals.

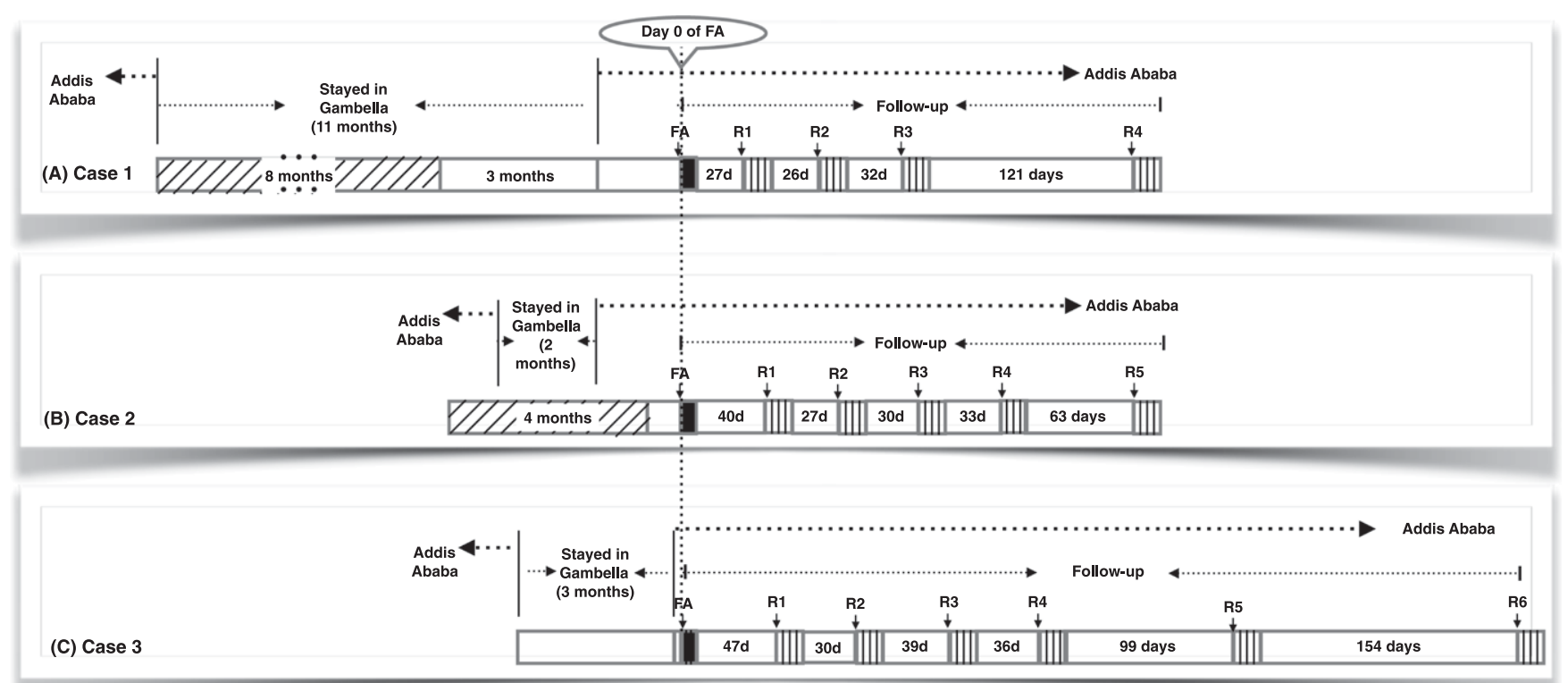

FA: First attack onset R\#: Relapse case onset $V / 7$ Chemoprophylaxis period

FA treatment period Relapse treatment period \#d Duration between relapses (days)

FIGURE 1. Timing of chloroquine prophylaxis, onset of primary and relapse attacks including treatment periods, interval between relapses, and time of travel to and from Gambella of the three cases, 1985-1987, Ethiopia. 

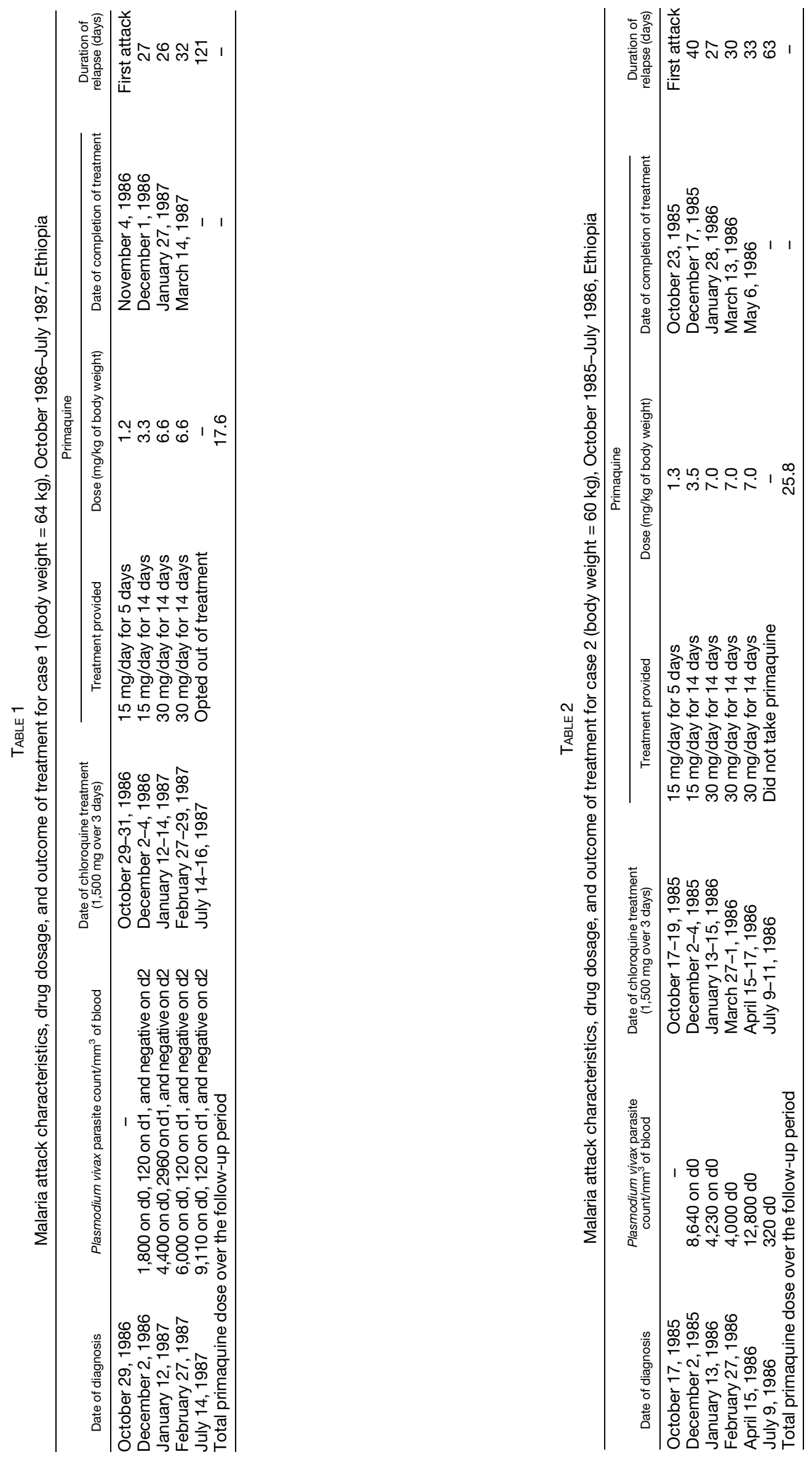


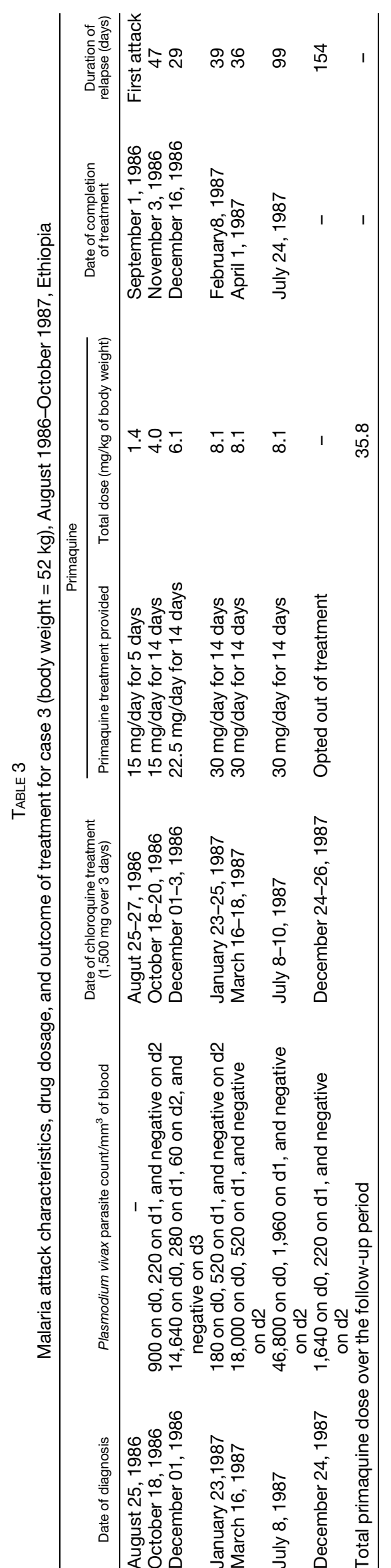

Blood smear examinations of the three cases during each visit had revealed the presence of $P$. vivax mono-infection parasites. They confirmed that they did not travel outside Addis Ababa, indicating that the three cases had relapse attacks. Considering that Addis Ababa is free of malaria transmission, the center treated the relapsing infections with chloroquine over 3 days followed by primaquine for 14 days based on WHO recommendations for radical cure as follows.

The first relapsing attack of case 1 was treated with a standard dose of $1,500 \mathrm{mg}$ base chloroquine over 3 days followed by $15 \mathrm{mg}$ base primaquine per day for 14 days. The subsequent two relapsing attacks were treated with a standard dose of $1,500 \mathrm{mg}$ base chloroquine over 3 days followed by $30 \mathrm{mg}$ base primaquine per day for 14 days each. Detailed information on parasite density, chloroquine and primaquine dose administered, and date of treatment completion, onset, and duration between relapses for the relapsing attacks is presented in Table 1. The total primaquine dose administered was $17.6 \mathrm{mg} / \mathrm{kg}$ of body weight. Similarly, all relapsing infections of case 2 were initially treated with a standard dose of $1,500 \mathrm{mg}$ base chloroquine over 3 days. Following the chloroquine treatment, the first relapsing attack was treated with $15 \mathrm{mg}$ base primaquine per day for 14 days. The subsequent three relapsing infections were treated with $30 \mathrm{mg}$ base per day for 14 days after completing their respective chloroquine regimens (Table 2). The total primaquine dose administered was $25.8 \mathrm{mg} / \mathrm{kg}$ of body weight. Relapsing infections of case 3 were also initially treated with a standard dose of $1,500 \mathrm{mg}$ base chloroquine over 3 days. Following the chloroquine treatment, the first two relapsing attacks were treated with $15 \mathrm{mg}$ base primaquine per day and $22.5 \mathrm{mg}$ base primaquine per day, respectively, each for 14 days. Each of the subsequent three relapses was treated with $30 \mathrm{mg}$ base primaquine per day for 14 days following the completion of the chloroquine regime (Table 3). Total primaquine dose administered was $35.8 \mathrm{mg} / \mathrm{kg}$ of body weight. The chloroquine treatment was efficacious as the asexual malaria parasites were cleared by day 2 in all first and relapsing infections, although $P$. falciparum resistance to chloroquine had been recorded more than three decades ago in Ethiopia. ${ }^{12}$

The patients were required to take their daily primaquine treatment doses at the Malaria Diagnostic Center under the supervision of laboratory personnel except for weekends when the drug was given to the patients with instruction to use them at home. Compliance for the self-treatment on weekends was ascertained by interview of the patients on following weekdays. Because primaquine can cause hemolytic anemia, particularly in those individuals who are deficient for the enzyme glucose-6-phosphate dehydrogenase (G6PD), ${ }^{13,14}$ routine testing for G6PD deficiency has to be carried out before initiating primaquine treatment for radical cure of $P$. vivax patients. However, because of lack of its availability, the three cases were not screened for G6PD deficiency. To ensure the safety of the patients, each patient taking repeated doses of primaquine was required to take hematology and clinical chemistry test at the National Research Institute of Health, which is now renamed as Ethiopian Public Health Institute. The treatments were well tolerated except for few complaints. Cases 1 and 2 had complained of abdominal cramp and tiredness, but each took their dosages and continued the follow-up for 8 and 9 months, respectively. Hematology and clinical chemistry test results of serum glutamic oxaloacetic transaminase, serum 
glutamic pyruvic transaminase, bilirubin, creatinine, albumin, protein, hemoglobin, and hematocrit for the three cases are summarized in Table 4. Case 3, who experienced an elevated direct and total bilirubin levels $(0.85 \mathrm{mg} \%$ and $2.88 \mathrm{mg}$ $\%$, respectively) and low hematocrit level (39.1), opted out after 16 months of follow-up.

\section{DISCUSSION}

The $P$. vivax malaria strains of the three cases were acquired in Gambella, Ethiopia, and managed for the first attack as well as relapses in a non-malarious setting in Addis Ababa, Ethiopia. The total doses of primaquine administered to cases 1, 2, and 3 were $17.6 \mathrm{mg} / \mathrm{kg}, 25.8 \mathrm{mg} / \mathrm{kg}$, and $35.8 \mathrm{mg} / \mathrm{kg}$, respectively, but failed to radically clear the parasites. The primaquine doses that failed to radically cure $P$. vivax infections from Gambella are much higher than doses previously reported with primaquine treatment failures for Ethiopia and East Africa ${ }^{10,11}$ but appear to fall within the category of primaquine doses recommended for Southeast Asia and Oceania. ${ }^{9,15}$ It also appears that the Gambella $P$. vivax malaria strain shows similar characteristics to $P$. vivax malaria from Southeast Asia in terms of early and frequent relapses and requirement for higher dosing of primaquine therapy. ${ }^{5,6}$ Because of multiple reports of primaquine failure for radical cure of $P$. vivax infections from various regions, the WHO recommends a primaquine dose of $15 \mathrm{mg}$ base daily for 14 days for all regions except for Southeast Asian region, which is recommended a dose of $30 \mathrm{mg}$ daily for 14 days. ${ }^{9}$ Nevertheless, the Centers for Disease Control (CDC) recommended use of $30 \mathrm{mg}$ daily for 14 days for all regions. ${ }^{16}$ The three cases from Ethiopia support other reports indicating failure of $P$. vivax malaria to respond to standard doses of primaquine in East Africa. ${ }^{17,18}$ A study on Israel tourists infected with $P$. vivax strain from Ethiopia contracted mostly from around the Omo River Valley showed that a primaquine dose $>3.5 \mathrm{mg} / \mathrm{kg}$ was effective in relapse prevention; however, the authors did not indicate the end point of the effective dose for radical cure. ${ }^{10}$

The contribution of polymorphisms in the human cytochrome P450 2D6 (CYP2D6) in explaining the findings can not be ruled out as its role in metabolizing primaquine to its redox-active metabolite and its association with primaquine failure is well documented. ${ }^{19-21}$ Although information relating to CYP2D activity in mediating primaquine metabolism for these three cases is not available, a study on the effect of CYP2D6 activity on the metabolism of debrisoquine among healthy Ethiopian population showed that $29 \%$ of the study participants carried duplicated and multiduplicated genes indicating ultrarapid metabolizers. ${ }^{22}$

Primaquine failure for radical cure of $P$. vivax malaria is a challenge to malaria control and elimination. The Ethiopian NMCP had developed a strategic plan for malaria elimination and updated its policy on primaquine use for radical treatment of $P$. vivax in selected districts targeted for malaria elimination and for those $P$. vivax patients who live outside malariaendemic areas. The information generated from the three cases relating to primaquine failure and characteristics of the relapsing episodes is of relevance to NMCP. ${ }^{13}$ The high-dose primaquine treatments were well tolerated except for few complaints and one case of elevated bilirubin level and low hematocrit level, indicating that the three cases were most probably not G6PD deficient. The total primaquine doses for cases 1 and 2 are in line with a previous observation that daily doses of $0.5 \mathrm{mg} / \mathrm{kg}$ for 50 weeks were well tolerated ${ }^{20}$ The prevalence of G6PD deficiency and its distribution in Ethiopia is not well known. ${ }^{23-25}$ Studies conducted in different settings in Ethiopia had indicated a low prevalence of G6PD deficiency in most parts of the country with pockets of higher prevalence in areas such as Gambella among the Nuer/Anuak population. ${ }^{23-25}$ The nation-wide study conducted by Assefa indicated the absence of the common G6PD-deficient African and Mediterranean variants. ${ }^{23}$

The case series analysis has some limitations. Primarily, the data are old, and thus, the efficacy of the drug may have changed over the years to reflect the current situation. However, primaquine has rarely been used for radical treatment in endemic areas of the country. In the absence of primaquine pressure for decades in the Ethiopian setting, it is unlikely that the efficacy of the drug as anti-relapse therapy of $P$. vivax has changed over time. Second, the data are based on the clinical records of patients who sought diagnosis and treatment as part of routine healthcare services and was not part of research-based investigation. Although this may affect the quality of the data, the procedure in service delivery and documentation of information that was followed ensured the availability of detailed and complete information on clinical presentation, treatment, and outcome. Because the case series is based on few cases from one site, the findings cannot be generalized and should be supported with other studies covering different geographic areas. Finally, future primaquine efficacy assessments for radical cure of $P$. vivax need to consider the role of CYP2D6 enzyme activity on the metabolism of primaquine to its active metabolites.

TABLE 4

Hematology and clinical chemistry test results of the three Plasmodium vivax cases by the series of relapse attacks, National Public Health Institute of Ethiopia, 1986-1987

\begin{tabular}{|c|c|c|c|c|c|c|c|}
\hline \multirow[b]{3}{*}{ Type of laboratory tests } & \multicolumn{7}{|c|}{ Date of laboratory testing (timing in relation to relapse attach series) } \\
\hline & \multicolumn{2}{|c|}{ Case 1} & \multicolumn{2}{|c|}{ Case 2} & \multicolumn{3}{|c|}{ Case 3} \\
\hline & $\begin{array}{c}\text { January 15, } 1987 \\
\text { (relapse 2) }\end{array}$ & $\begin{array}{l}\text { March 20, } 1987 \\
\quad \text { (relapse 3) }\end{array}$ & $\begin{array}{l}\text { January 4, } 1986 \\
\quad \text { (relapse 2) }\end{array}$ & $\begin{array}{l}\text { May 10, 1986 } \\
\text { (relapse 4) }\end{array}$ & $\begin{array}{l}\text { December 6, } 1986 \\
\quad \text { (relapse 2) }\end{array}$ & $\begin{array}{l}\text { July } 13,1987 \\
\text { (relapse 4) }\end{array}$ & $\begin{array}{c}\text { December 27, } 1987 \\
\text { (relapse 6) }\end{array}$ \\
\hline SGOT (IU/L) & 17 & 21 & 15 & 21 & 26 & 22 & 26 \\
\hline SGPT (IU/L) & 35 & 33 & 23 & 35 & 24 & 22 & 32 \\
\hline Bilirubin direct (mg/dL) & 0.12 & 0.51 & 0.03 & 0.24 & 0.40 & 0.45 & $0.85^{\star}$ \\
\hline Bilirubin total (mg/dL) & 0.82 & 1.52 & 0.62 & 0.49 & 1.40 & 1.41 & $2.88^{*}$ \\
\hline Creatinine (mg/dL) & 1.1 & 0.9 & 1.0 & 1.5 & 1.4 & 1.0 & 1.4 \\
\hline Protein (mg/dL) & 7.1 & 6.5 & 7.9 & - & 6.9 & 5.8 & 7.9 \\
\hline Albumin (mg/dL) & 3.8 & 5.0 & 4.2 & 4.5 & 3.8 & - & 4.0 \\
\hline Hemoglobin (gm) & 14.2 & 13.1 & 17 & 17 & 15.3 & 13.4 & 14 \\
\hline Hematocrit (\%) & 42 & 39 & 51 & 52 & 45 & - & $39.1^{*}$ \\
\hline
\end{tabular}




\section{CONCLUSION}

Primaquine failure for the radical cure of $P$. vivax malaria in Ethiopia is a challenge to malaria control and elimination in the country. The high-dose primaquine failure for treatment of the three patients observed during 8-16 months of follow-up is a concern that would demand systematic studies. Cytochrome P-450 2D6 is involved in the metabolism of primaquine to its active metabolite against hypnozoites, and it is important to identify the association between CYP2D6 activity and primaquine failure in different settings in Ethiopia. It will be prudent to consider adjusting the standard treatment dose of primaquine for radical cure to daily $0.5 \mathrm{mg} /$ $\mathrm{kg}$ for 14 days in-line with the $\mathrm{CDC}$ recommendation and the $\mathrm{WHO}$ guideline issued for Southeast Asia. Despite the high primaquine treatment regimen, the treatments were well tolerated. In view of the requirement for high primaquine dosing, $P$. vivax patients need to be tested for G6PD deficiency before initiating treatment with primaquine. As this case series is limited to Gambella region only, additional study in different geographical areas of the country is suggested to determine the prevalence and distribution of primaquine treatment failures to come up with appropriate national drug policy. Compliance on the use of 14-day primaquine regimen is a challenge for malaria elimination in Ethiopia. It has been reported that use of a single dose of 300-600 mg base of tafenoquine and chloroquine combination is as efficacious as primaquine $15 \mathrm{mg} / \mathrm{kg} /$ day for 14 days for preventing relapse of $P$. vivax. ${ }^{26}$ We recommend that such combination be evaluated in an Ethiopian setting.

Received December 2, 2019. Accepted for publication April 6, 2020.

Published online May 11, 2020.

Acknowledgments: We gratefully acknowledge the input of professionals who provided expert opinion in undertaking the case report. We would like to thank the National Malaria Laboratory Center, Addis Ababa, for availing the records of the cases.

Financial support: A. T. and H. D. T. were supported by CNHDE. Saint Paulos Hospital, Millennium Collage, and National Research Institute of Health supported A. G. and A. W., respectively.

Disclaimer: The funders had no role in the study design, data analysis, data interpretation, or writing of the manuscript.

Authors' addresses: Awash Teklehaimanot and Hailay Teklehaimanot, Centers for National Health Development Ethiopia, Addis Ababa, Ethiopia, E-mails: athbruk1@gmail.com and hdteklehaim@gmail.com. Abeba Girmay, Laboratory Department, Saint Paulos Hospital, Millennium Collage, Addis Ababa, Ethiopia, E-mail: lozaberhane@yahoo. com. Adugna Woyessa, National Research Institute of Health, Addis Ababa, Ethiopia, E-mail: adugnawoyessa@gmail.com.

\section{REFERENCES}

1. Federal Ministry of Health of Ethiopia, 2012. National Malaria Guidelines, 3rd edition. Addis Ababa, Ethiopia: FMOH.

2. Federal Ministry of Health of Ethiopia, 2015. Health and Health Related Indicators. annual edition. Addis Ababa, Ethiopia: $\mathrm{FMOH}$.

3. Wells TNC, Burrows JN, Baird JK, 2010. Targeting the hypnozoite reservoir of Plasmodium vivax: the hidden obstacle to malaria elimination. Trends Parasitol 26: 145-151.

4. Goller JL, Jolley D, Ringwald P, Biggs BA, 2007. Regional differences in the response of Plasmodium vivax malaria to primaquine as anti-relapse therapy. Am J Trop Med Hyg 76: 203-207.

5. John GK, Douglas NM, Von Seidlein L, Nosten F, Baird JK, White NJ, Price RN, 2012. Primaquine radical cure of Plasmodium vivax: a critical review of the literature. Malar J 11: 280.
6. White NJ, 2011. Determinants of relapse periodicity in Plasmodium vivax malaria. Malar J 10: 297.

7. Noviyanti R et al., 2015. Contrasting transmission dynamics of coendemic Plasmodium vivax and $P$. falciparum: implications for malaria control and elimination. PLoS Negl Trop Dis 9: e0003739.

8. Ministry of Health of the Federal Democtratic Republic of Ethiopia, 2010. National Strategic Plan for Malaria Prevention Control and Elimination in Ethiopia 2011-2015. Addis Ababa, Ethiopia: $\mathrm{FMOH}$.

9. World Health Organization, 2015. Guidelines For The Treatment of Malaria, 3rd edition. Geneva, Switzerland: WHO.

10. Schwartz E, Regev-Yochay G, Kurnik D, 2000. Short report: a consideration of primaquine dose adjustment for radical cure of Plasmodium vivax malaria. Am J Trop Med Hyg 62: 393-395.

11. Smoak BL, DeFraites RF, Magill AJ, Kain KC, Wellde BT, 1997. Plasmodium vivax infections in U.S. army troops: failure of primaquine to prevent relapse in studies from Somalia. $A m \mathrm{~J}$ Trop Med Hyg 56: 231-234.

12. Teklehaimanot $A, 1986$. Chloroquine-resistant Plasmodium falciparum malaria in Ethiopia. Lancet 2: 127-129.

13. Recht J, Ashley EA, White NJ, 2018. Use of primaquine and glucose-6-phosphate dehydrogenase deficiency testing: divergent policies and practices in malaria endemic countries. PLoS Negl Trop Dis 12: e0006230.

14. Baird JK, Battle KE, Howes RE, 2018. Primaquine ineligibility in anti-relapse therapy of Plasmodium vivax malaria: the problem of G6PD deficiency and cytochrome P-450 2D6 polymorphisms. Malar J 17: 42.

15. Fernando D, Rodrigo C, RajapakseS, 2011. Primaquine in vivax malaria: an update and review on management issues. Malar J 10: 351.

16. Hill DR, Baird JK, Parise ME, Lewis LS, Ryan ET, Magill AJ, 2006. Primaquine: report from CDC expert meeting on malaria chemoprophylaxis I. Am J Trop Med Hyg 75: 402-415.

17. Abreha T et al., 2017. Comparison of artemether-lumefantrine and chloroquine with and without primaquine for the treatment of Plasmodium vivax infection in Ethiopia: a randomized controlled trial. PLoS Med 14: e1002299.

18. Yeshiwondim AK, Tekle AH, Dengela DO, Yohannes AM, Teklehaimanot A, 2010. Therapeutic efficacy of chloroquine and chloroquine plus primaquine for the treatment of Plasmodium vivax in Ethiopia. Acta Trop 113: 105-113.

19. Bennett JW, Pybus BS, Yadava A, Tosh D, Sousa JC, McCarthy WF, Deye G, Melendez V, Ockenhouse CF, 2013. Primaquine failure and cytochrome P-4502D6 in Plasmodium vivax malaria. N Engl J Med 369: 1381-1382.

20. Baird JK et al., 2018. Association of impaired cytochrome P450 2D6 activity genotype and phenotype with therapeutic efficacy of primaquine treatment for latent Plasmodium vivax malaria. JAMA Netw Open 1: e181449.

21. Rajman I, Knapp L, Morgan T, Masimirembwa C, 2017. African genetic diversity: implications for cytochrome P450-mediated drug metabolism and drug development. EBioMedicine 17: 67-74.

22. Aklillu E, Persson I, Bertilsson L, Johansson I, Rodrigues F, Ingelman-Sundberg M, 1996. Frequent distribution of ultrarapid metabolizers of debrisoquine in an Ethiopian population carrying duplicated and multiduplicated functional CYP2D6 alleles. J Pharmacol Exp Ther 278: 441-446.

23. Assefa A et al., 2018. Glucose-6-phosphate dehydrogenase (G6PD) deficiency in Ethiopia: absence of common African and Mediterranean allelic variants in a nationwide study. Malar J 17: 388.

24. Shitaye $\mathrm{G}$ et al., 2018. Low and heterogeneous prevalence of glucose-6-phosphate dehydrogenase deficiency in different settings in Ethiopia using phenotyping and genotyping approaches. Malar J 17: 281.

25. Tsegaye A, Golassa L, Mamo H, Erko B, 2014. Glucose-6phosphate dehydrogenase deficiency among malaria suspects attending Gambella hospital, southwest Ethiopia. Malar J 13: 348.

26. Chu CS, White NJ, Chu CS, 2016. Management of relapsing Plasmodium vivax malaria. Expert Rev Anti Infect Ther 14: 885-900. 